\title{
Optical eigenmode description of partially coherent light fields
}

\section{Michael Mazilu}

Michael Mazilu, "Optical eigenmode description of partially coherent light fields," Proc. SPIE 10935, Complex Light and Optical Forces XIII, 1093508 (4 March 2019); doi: 10.1117/12.2507901

SPIE. Event: SPIE OPTO, 2019, San Francisco, California, United States 


\title{
Optical eigenmode description of partially coherent light fields
}

\author{
Michael Mazilu \\ SUPA, School of Physics and Astronomy, \\ University of St Andrews, St Andrews, United Kingdom
}

\begin{abstract}
Optical eigenmodes describe coherent solutions of Maxwells equations that are orthogonal to each other. These modes form a natural basis set of the electromagnetic Hilbert space that can be used to describe optical scattering interactions in a simple way. Many of the properties defined in quantum mechanics can formally be found in the optical eigenmodes framework. For example, the Hilbert spaces defined by two different scattering operators are separable only if the two operators commute with each other. Here, we expand the optical eigenmode framework to partially coherent light fields. In this case, we remark that the eigenmode decomposition of partially coherent fields leads to a formalism similar to the density matrix formalism used in quantum mechanics.
\end{abstract}

Keywords: Optical eigenmodes, partial coherence

\section{INTRODUCTION}

Scattering is one of the simplest light mater interactions possible. For spherical particles, this process can be described using the Lorenz-Mie theory, which makes use of vector spherical harmonic solutions of Maxwells equations to represent the fields involved. Using these solutions it is possible to describe the light field scattered from microscopic spherical particles and thus represent the field around a scattering object as a function of the incident fields. These solutions also allow us to determine the optical momentum transfer to the scattering object. This can be calculated using Maxwell stress tensor. Here, we will study the momentum transfer to a spherical particle illuminated by a superposition of vector Bessel beams and introduce the use of optical eigemodes to describe the optical forces acting of the microsphere. These eigenmodes are then used to assess the effect of the scattering on the coherence of the incident light field.

"The decomposition of fields into eigenmodes is a common technique to solve various problems. Such a decomposition can be used to describe the propagation of light in a variety of setups such as waveguides, photonic crystals, and optical cavities. The method of Optical Eigenmodes (OEi) has been introduced as a generalization of this mode decomposition going beyond describing the propagation properties. ${ }^{1}$ This method applies to any quadratic measure of the electromagnetic field such as energy density, momentum, angular momentum, etc. ${ }^{2,3}$ In addition, we can describe the change in these quantities when interacting with matter, giving the forces or torques experienced by particles. This method consists of finding the eigenmodes of the quadratic measure in question. These input light fields contribute to the measure independently, without interference, allowing for example the addressing of individual plasmonic nano-antennas of an array without cross-talk. ${ }^{4}$ It is worth noting that as this method results in a set of eigenmodes which contribute an amount equal to the corresponding real eigenvalue, optimisation of a quantity such as spot size or energy transmission is as trivial as choosing the mode with the highest or lowest eigenvalue."

Send correspondence to: mm17@st-andrews.ac.uk 


\section{OPTICAL EIGENMODES FOR PARTIALLY COHERENT FIELDS}

\subsection{Optical eigenmode definition}

"Expanding the electric field as a superposition of some basis fields $\mathbf{E}=\sum_{i} \alpha_{i} \mathbf{E}_{i}$, we can write a general quadratic function of the fields as a Hermitian form of these coefficients $m(\mathbf{E})=\alpha^{*} \mathcal{M} \alpha$ where $\mathcal{M}$ is a Hermitian matrix. For the expansion to be exact we require a complete set of basis fields; in practice we can keep only a finite number of basis functions which span a subspace, for example a set of low-order Hermite-Gauss beams. Since $\mathcal{M}$ is Hermitian, it has a spectrum of real eigenvalues which can be ordered to find the maximum or minimum possible value of the quantity of interest and the corresponding optical eigenmode which produces it."

The optical eigenmodes are defined as:

$$
\begin{aligned}
\mathbb{E}_{\lambda} & =\sum_{i} a_{\lambda i} \mathbf{E}_{i}, \\
\lambda \mathbf{a}_{\lambda} & =\mathcal{M} \mathbf{a}_{\lambda} .
\end{aligned}
$$

\subsection{Partially coherent fields}

Any incident field can be decompose on the optical eigenmode basis:

$$
\mathbf{E}=\sum_{j} c_{j} \mathbb{E}_{j}
$$

In this case a generic quadratic measure is defined by

$$
m=c_{i}^{*} M_{i j} c_{j}
$$

where $M$ is the Hermitian matrix associated with the measurement. When the optical eigenmodes are those of the quadratic measure $M_{i j}$ that we have $M_{i j}=m_{\lambda i} \delta_{i j}$ where $m_{\lambda i}$ is the eigenvalue associated with $\mathbb{E}_{i}$.

For partially coherent light fields, we consider a stochastic distribution of multiple fields $\mathbf{E}_{k}=\sum_{j} c_{k j} \mathbb{E}_{j}$ with probabilities $p_{k}$ such that $\sum_{k} p_{k}=1$ with $p_{k}>0$. In this case, we can define the mean measure as

$$
<m>=\sum_{k} p_{k} m_{k}=\sum_{k} p_{k} c_{k i}^{*} M_{i j} c_{k j}=\operatorname{Tr}(\rho M)
$$

where $\rho=\sum_{k} p_{k} \rho_{k}$ with $\rho_{k}=c_{k i}^{*} c_{k j}$. This definition is similar to the quantum mechanic treatment of the density matrix formalism. ${ }^{5}$

\section{APPLICATION: MOMENTUM TRANSFER TO MICROPARTICLES}

In the following, we work with monochromatic fields $\left(\omega=k_{0} c\right)$ which define the incident field as a superposition of Bessel beams that are either azimuthally (s-polarisation) or radially polarised (p-polarisation). Each Bessel beam is further characterised by its transversal wavevector $k_{t}=n_{0} k_{0} \sin (\gamma)$ and longitudinal wavevector $k_{z}=$ $n_{0} k_{0} \cos (\gamma)$ where $\gamma$ and $n_{0}$ are the cone half-angle of the Bessel beam and index of refraction of the propagation media, respectively.

In a first step, we define the vector Bessel beams in Cartesian coordinate system and their radial component in the spherical coordinate system. This component allows us to define the beam shape coefficients that together with the momentum transfer operator can be used to calculate the optical forces acting on spherical particles. The eigenmodes of the momentum operator can then be introduced, reducing the dimensionally of the system when calculating the momentum transfer. 


\subsection{Vector Bessel beam shape coefficients}

To simplify the definition of the Bessel beam in Cartesian coordinates, we introduce the following function:

$$
B_{\ell}\left(k_{t} \rho\right)=(i)^{\ell} J_{\ell}\left(k_{t} \rho\right) e^{i \ell \phi}
$$

with $\rho=\sqrt{x^{2}+y^{2}}$ and $\phi=\arctan (y / x)$ and where $J_{\ell}(r)$ are the Bessel functions of the first kind.

In this case, the s-polarised Bessel beam takes the form:

$$
\begin{gathered}
\mathbf{E}^{\ell(s)}=\left(\begin{array}{c}
E_{x}^{\ell(s)} \\
E_{y}^{\ell(s)} \\
E_{z}^{\ell(s)}
\end{array}\right)=c_{w}\left(\begin{array}{l}
-i B_{\ell-1}\left(k_{t} \rho\right)+i B_{\ell+1}\left(k_{t} \rho\right) \\
B_{\ell-1}\left(k_{t} \rho\right)+B_{\ell+1}\left(k_{t} \rho\right) \\
0
\end{array}\right) \\
\mathbf{H}^{\ell(s)}=\left(\begin{array}{c}
H_{x}^{\ell(s)} \\
H_{y}^{\ell(s)} \\
H_{z}^{\ell(s)}
\end{array}\right)=c_{w} \frac{n}{c \mu_{0}} \cos (\gamma)\left(\begin{array}{l}
-B_{\ell-1}\left(k_{t} \rho\right)-B_{\ell+1}\left(k_{t} \rho\right) \\
-i B_{\ell-1}\left(k_{t} \rho\right)+i B_{\ell+1}\left(k_{t} \rho\right) \\
2 B_{\ell}\left(k_{t} \rho\right) \tan (\gamma)
\end{array}\right)
\end{gathered}
$$

where the $c_{w}=\pi \sin (\gamma) \sqrt{\cos (\gamma)} \exp \left(-i \omega t+i k_{z} z\right)$.

The p-polarised beam can be related to the s-polarised one via: $\mathbf{E}^{\ell(p)}=-c \mu_{0} / n \mathbf{H}^{\ell(s)}$ and $\mathbf{H}^{\ell(p)}=n /\left(c \mu_{0}\right) \mathbf{E}^{\ell(s)}$. The main property of these Bessel beams is that they are eigenfunctions of the solid rotation operator with a integer valued eigenvalue $\ell$

$$
\begin{aligned}
-i \mathbf{e}_{z} \times \mathbf{E}^{\ell(s, p)}-i(\mathbf{r} \times \nabla)_{\mathbf{e}_{z}} \mathbf{E}^{\ell(s, p)} & =\ell \mathbf{E}^{\ell(s, p)} \\
-i \mathbf{e}_{z} \times \mathbf{H}^{\ell(s, p)}-i(\mathbf{r} \times \nabla)_{\mathbf{e}_{z}} \mathbf{H}^{\ell(s, p)} & =\ell \mathbf{H}^{\ell(s, p)}
\end{aligned}
$$

where $\mathbf{e}_{z}$ is the z-direction unit vector. The two terms in this operator can be identified with the spin angular momentum and the orbital angular momentum, respectively.

Projecting the electric and magnetic fields on the radial unit vector in spherical coordinates defines the radial components of the electromagnetic fields as

$$
\begin{aligned}
E_{r}^{\ell(s)}(r, \theta, \phi)= & i^{\ell} c_{s} \exp (i \ell \phi)\left(-J_{\ell-1}\left(k_{t} r \sin \theta\right) \sin \theta-J_{\ell+1}\left(k_{t} r \sin \theta\right) \sin \theta\right) \\
H_{r}^{\ell(s)}(r, \theta, \phi)= & i^{\ell} c_{s} \frac{n}{c \mu_{0}} \exp (i \ell \phi) \\
& \left(2 J_{\ell}\left(k_{t} r \sin \theta\right) \cos \theta \sin \gamma+i J_{\ell-1}\left(k_{t} r \sin \theta\right) \sin \theta \cos \gamma-i J_{\ell+1}\left(k_{t} r \sin \theta\right) \sin \theta \cos \gamma\right)
\end{aligned}
$$

where $c_{s}=\pi \sin (\gamma) \sqrt{\cos (\gamma)} \exp \left(-i \omega t+i k_{z} z \cos (\theta)\right)$

The beam shape coefficients can then be determined by calculating the inner product of the radial field component with the spherical harmonic function $Y_{n}^{m}(\theta, \phi)$

$$
j_{n}(k r)\left(\begin{array}{c}
-g_{n m}^{T M} \\
g_{n m}^{T E} / Z
\end{array}\right)=\frac{k r}{\sqrt{n(n+1)}} \int_{0}^{\pi} d \theta \int_{0}^{2 \pi} d \phi Y_{n}^{m}(\theta, \phi)^{*}\left(\begin{array}{c}
E_{r} \\
H_{r}
\end{array}\right)
$$

with $Z=\mu_{0} c / n_{0}$ and where $*$ stands for the complex conjugate and where $g_{n m}^{T M}$ and $g_{n m}^{T E}$ are the beam shape coefficients.

Introducing $c_{m}=\pi \sin (\gamma) \sqrt{\cos (\gamma)} \exp (-i \omega t)$, we have for the s-polarised Bessel beam

$$
\begin{aligned}
g_{n m}^{T M} & =\delta_{m}^{\ell} \frac{4 \pi i^{n} \sqrt{\pi \cos \gamma(2 n+1)(n-m) !}}{\sqrt{n(n+1)(n+m) !}}(m) P_{n}^{m}(\cos \gamma) \\
g_{n m}^{T E} & =\delta_{m}^{\ell} \frac{4 \pi i^{n} \sqrt{\pi \cos \gamma(2 n+1)(n-m) !}}{\sqrt{n(n+1)(n+m) !}}(-i)\left((n+1) \cos \gamma P_{n}^{m}(\cos \gamma)+(m-n-1) P_{n+1}^{m}(\cos \gamma)\right)
\end{aligned}
$$


valid for $n \geq \ell$. In the following, we define a single list of beam shape coefficients

$$
g_{j}=g_{n_{j} m_{j}}^{p_{j}}
$$

where $m_{j}, n_{j}$ and $p_{j}$ are all coefficients considered indexed using the subscript $j$. The polarisation index $p_{k}$ can take the values of 1 and 2 corresponding respectively to $T M$ and $T E$ modes. A superposition of vector Bessel beams will then correspond to a superposition of beam shape coefficients $g_{i}$. Indeed, an angular dependent superposition of Bessel beams defined this way makes it possible to describe high-NA beams used in microscopes which introduce spherical aberrations. ${ }^{6}$

\subsection{Momentum transfer}

Momentum transfer to scattering objects can be calculated using Maxwell's stress tensor defined by

$$
\widetilde{\sigma}=\frac{1}{2}\left(\left(n_{0}^{2} \epsilon_{0} \mathbf{E} \cdot \mathbf{E}+\mu_{0} \mathbf{H} \cdot \mathbf{H}\right) \widetilde{I}-2 n_{0}^{2} \epsilon_{0} \mathbf{E} \otimes \mathbf{E}-2 \mu_{0} \mathbf{H} \otimes \mathbf{H}\right),
$$

where $\otimes$ stands for the tensor product and $\widetilde{I}$ for the identity $3 \times 3$ matrix. In a first instance, we are interested in the force in the $z$-direction which can be calculated by integrating over a spherical surface $S$ surrounding the object

$$
F_{z}=<\oint_{S} \mathbf{e}_{z} \tilde{\sigma} d \mathbf{n}>
$$

where $<>$ stands for the time average over the optical cycle. The fields $\mathbf{E}$ and $\mathbf{H}$ correspond to the sum of the incident and scattered fields. The incident fields are defined by the beam shape coefficients while the scattered fields can be calculated using the Mie scattering coefficients and the beam shape coefficients. Altogether, we remark that the optical force acting in the z-direction can be expressed in a quadratic form with respect to the beam shape coefficients. This quadratic Hermitian form is based on the matrix operator

$$
\begin{aligned}
M_{k}^{j} & =\frac{1}{2} \delta_{m_{k}}^{m_{j}} \delta_{p_{k}}^{p_{j}}\left(\delta_{n_{k}}^{n_{j}+1} \sqrt{\frac{\left(1-n_{k}\right)^{2}\left(n_{j}^{2}-m_{k}^{2}\right)}{n_{k}^{2}\left(4 n_{k}^{2}-1\right)}}-\delta_{n_{k}+1}^{n_{j}} \sqrt{\frac{\left(1-n_{j}\right)^{2}\left(n_{k}^{2}-m_{k}^{2}\right)}{n_{j}^{2}\left(4 n_{j}^{2}-1\right)}}\right) \\
& \left(\delta_{p_{k}}^{1}\left(b_{k}+b_{j}^{*}-2 b_{k} b_{j}^{*}\right)+\delta_{p_{k}}^{2}\left(a_{k}+a_{j}^{*}-2 a_{k} a_{j}^{*}\right)\right) \\
& +\frac{i}{2} \delta_{n_{k}}^{n_{j}} \delta_{m_{k}}^{m_{j}} \frac{m_{k}}{n_{k}\left(n_{k}+1\right)}\left(\delta_{p_{k}}^{1} \delta_{2}^{p_{j}}\left(b_{k}+a_{k}^{*}-2 b_{k} a_{k}^{*}\right)-\delta_{p_{k}}^{2} \delta_{1}^{p_{j}}\left(a_{k}+b_{k}^{*}-2 a_{k} b_{k}^{*}\right)\right)
\end{aligned}
$$

where $n_{k} m_{k}$ and $p_{k}$ is the $n$ - and $m$-index and polarisation state of the $k$-th beam shape component and where $a_{k}$ and $b_{k}$ are the $n_{k}$-indexed Mie scattering coefficients of the scattering object.

The optical momentum transfer in the z-direction is then defined by

$$
F_{z}=g^{k} M_{k}^{j} g_{j}
$$

where $g^{k}=g_{k}^{*}$. This quadratic Hermitian expression calculates the force acting in the z-direction on the scattering object positioned in at the origin of the coordinate system however the direction can be changed using spherical harmonics rotation and translation matrices.

\subsection{Optical eigenmodes}

The momentum transfer matrix is by construction Hermitian and in general all real field properties that can be expressed as a function of field in a quadratic form will lead to a matrix/operator that is Hermitian. ${ }^{7,8}$ The consequence of this observation is that the momentum transfer matrix defines a set of orthogonal vectors that correspond to the eigenvectors of $M_{k}^{j}$. Each of these eigenvectors defines a field that we call optical eigenmode of the measure in questions, in this case it is the optical eigenmode of $F_{z}$. If the Mie scattering order tends to infinity then the optical eigenmodes will form a complete Hilbert basis set of solutions of Maxwell's equations. Each quadratic measure will define an operator with its own set of optical eigenmodes. If two operators commute then it is possible to define a set of optical eigenmodes that are simultaneously eigenmodes for each operator. 

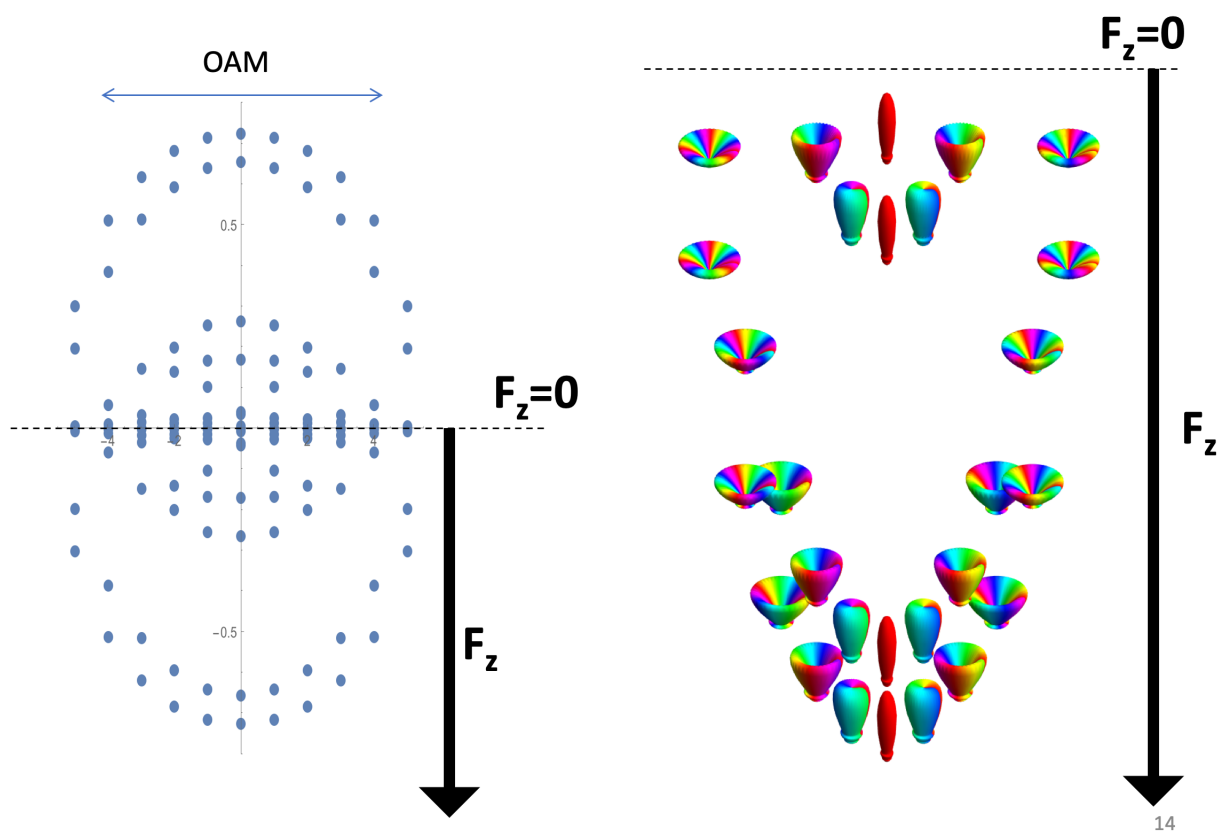

Figure 1. (a) Eigenvalue representation of the bound momentum (linear $F_{z}$ and orbital $L_{z}$ ) optical eigenmodes. (b) Angular intensity distribution of the optical eigenmodes. The colour indicate the phase of the field.
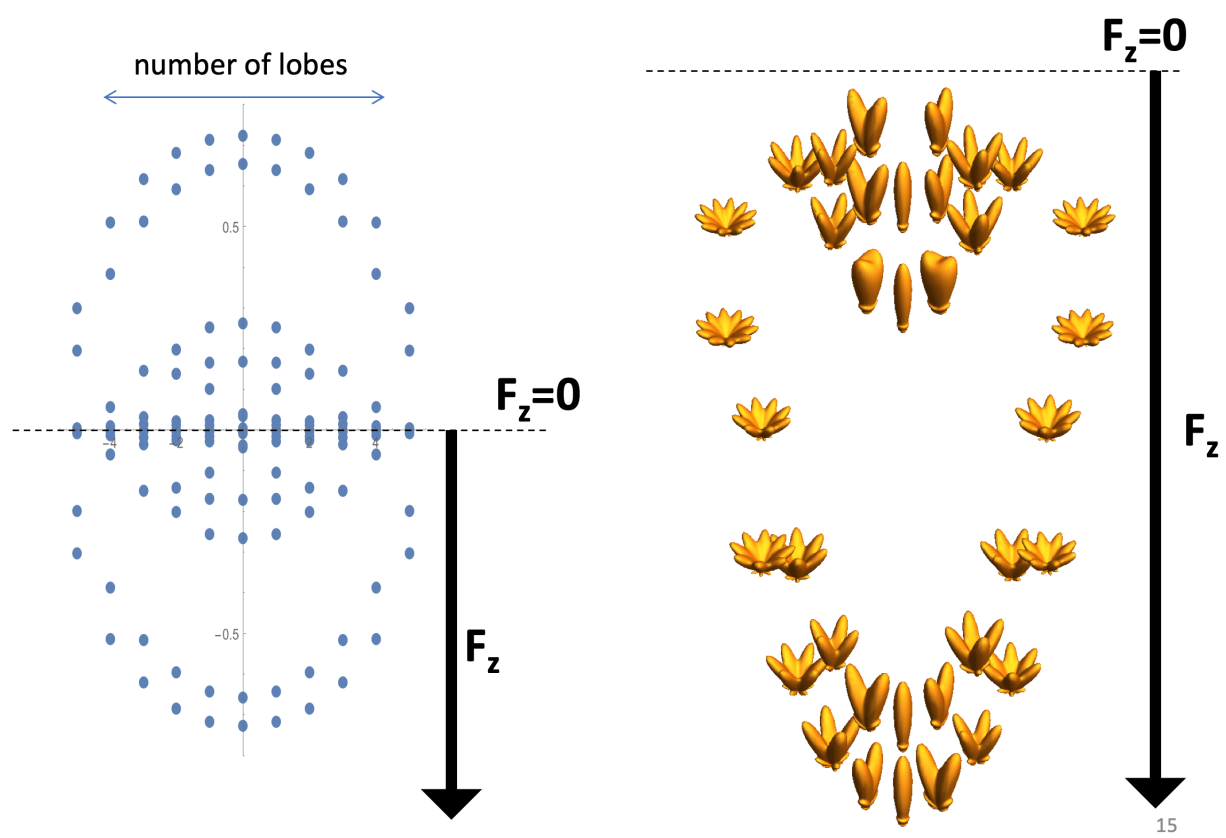

Figure 2. (a) Eigenvalue representation of the bound linear momentum, $F_{z}$, optical eigenmodes. The horizontal axis counts the number of lobes and their position. (b) Angular intensity distribution of the optical eigenmodes. Note, the fields are real and the is no orbital angular momentum. These modes are obtained by hybridisation of the modes in Fig.1. 


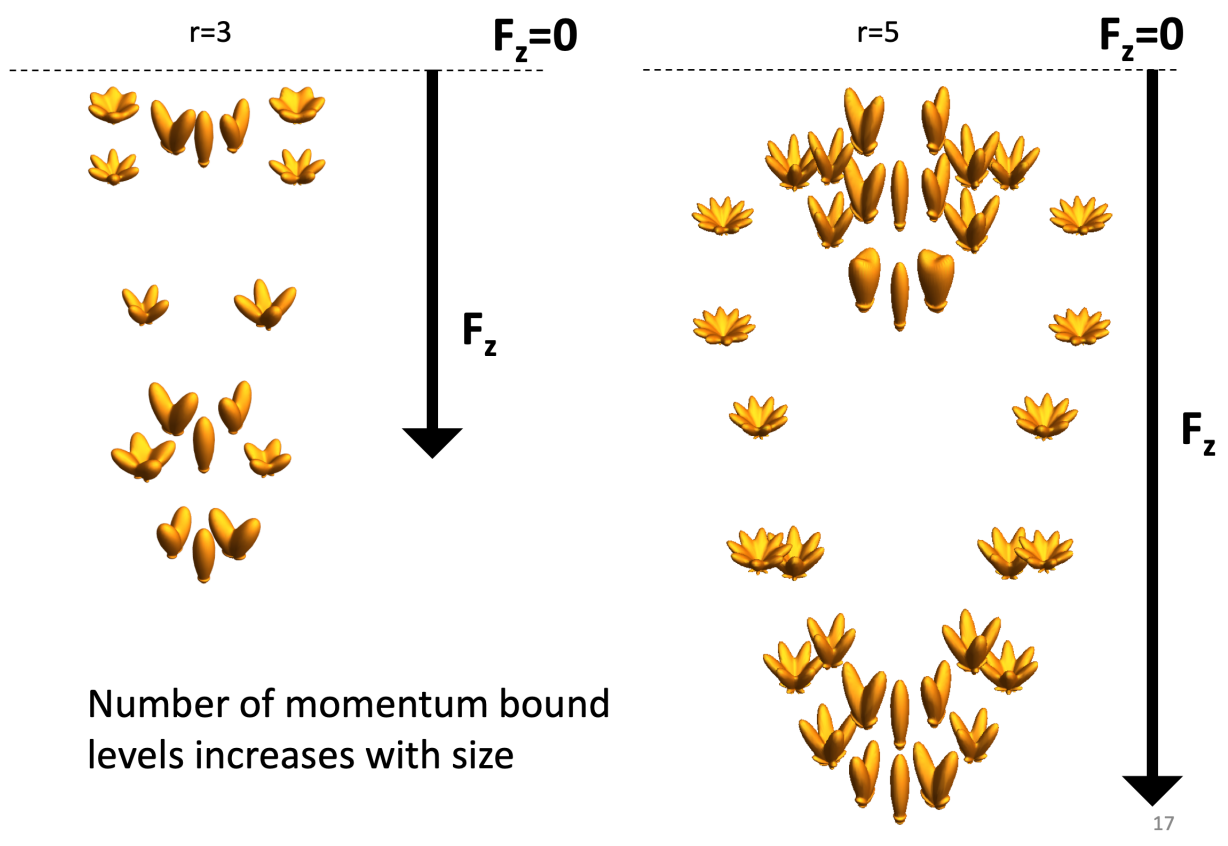

Figure 3. Angular intensity distribution of the optical eigenmodes for two particles of different sizes.

Each optical eigenmodes has a real eigenvalue associated with it. This value corresponds in this case to the optical force acting in the z-direction on the scattering object when the incident field is the optical eigenmode. Considering the set of all operators that commute with each other then the set of eigenvalues from these operators can be used to uniquely identify an optical field (see Figs. 1, 2 and 3).

Further, the eigenvalues can be used to sort the optical eigenmodes by order of importance such that we can reduce the degrees of freedom for which a system needs to be solved for by discarding all optical eigenmodes that are not contributing. We determine the optical eigenmodes that lead to any significant momentum transfer and use these modes to describe the incident field. Any incident field that does not couple to the optical eigenmodes will not lead to any measurable momentum transfer due to the symmetries of the problem considered.

\subsection{Transfer of momentum from partially coherent fields}

Here, we consider a monochromatic wave incident onto a Mie particles which measures the momentum transfer along the z-axis. Equation 5 implies that the probability to measure a specific eigenvalue $F_{z}$ is proportional to the norm squared of the overlap integral (projection coefficient) of the incident field onto the optical eigenmodes exemplified in Figures 1,2 and 3. A consequence of this definition if that the measure of momentum in a scattering event will lead to a change in partial coherence probability distribution of the scattered field. This change in distribution will depend on the optical eigenmodes and on other factors such as the mass of the particle.

\section{REFERENCES}

[1] Mazilu, M., Baumgartl, J., Kosmeier, S., and Dholakia, K., "Optical eigenmodes; exploiting the quadratic nature of the energy flux and of scattering interactions," Opt. Express 19, 933-945 (Jan 2011).

[2] Mazilu, M., "Spin and angular momentum operators and their conservation," J. Opt. A 11(9), 094005 (2009).

[3] Mazilu, M., "Optical eigenmodes; spin and angular momentum," J. Opt. 13(6), 064009 (2011).

[4] Kosmeier, S., De Luca, A. C., Zolotovskaya, S., Di Falco, A., Dholakia, K., and Mazilu, M., "Coherent control of plasmonic nanoantennas using optical eigenmodes," Scientific Reports 3, 1808 EP - (May 2013). Article.

[5] Cohen-Tannoudji, C., Diu, B., and Laloë, F., [Quantum mechanics], Hermann (1977). 
[6] Ranha Neves, A. A., Fontes, A., Cesar, C. L., Camposeo, A., Cingolani, R., and Pisignano, D., "Axial optical trapping efficiency through a dielectric interface," Phys. Rev. E 76, 061917 (2007).

[7] Baumgartl, J., Kosmeier, S., Mazilu, M., Rogers, E. T. F., Zheludev, N. I., and Dholakia, K., "Far field subwavelength focusing using optical eigenmodes," Appl. Phys. Lett. 98(18), 181109 (2011).

[8] De Luca, A. C., Kosmeier, S., Dholakia, K., and Mazilu, M., "Optical eigenmode imaging," Phys Rev A 84(2), 021803(R) (2011). 